\title{
Detection of Brain Diseases using EEG and Speech Signal
}

\author{
Akshata S. Agarwal \\ M.Tech Student \\ Department of Electronics Engg. \\ Vishwakarma Institute of Technology, \\ Pune-037
}

\author{
Kishori S. Degaonkar \\ Associate Professor \\ Department of Electronics Engg. \\ Vishwakarma Institute of Technology, \\ Pune-037
}

\begin{abstract}
Parkinson's disease (PD) and Alzheimer's diseases are the most common brain diseases. Parkinson's disease (PD) occurs when the neurons that produce dopamine in the brain are damaged. People aged 50 or above mostly suffer from Parkinson's disease. PD and Alzheimer's disease can be diagnosed by many different signals such as EEG and Speech signals. This paper proposes a method for detecting PD and Alzheimer's disease where, discrete wavelet transform feature extraction technique were used and SVM network is used for classification. The accuracy of $91.6 \%$ is obtained.
\end{abstract}

\section{Keywords}

Parkinson's disease, Alzheimer disease, EEG signals, speech, SVM

\section{INTRODUCTION}

In medical field, brain signals have been studied and observed to diagnosis and detect different brain diseases like Parkinson's, Alzheimer's, schizophrenia, epilepsy, Aphasia.[3] Electroencephalography (EEG) signal is a method to monitor and record electrical activities of the brain which is placed along the scalp. EEG measures fluctuations in the voltage within the neurons of the brains

Parkinson disease (PD) was discovered by James Parkinson's in London in 1817 for the first time. PD is the second neurodegenerative disease after Alzheimer disease. People suffering from PD are aged 50 or above and 4 to 6 million people suffer it worldwide. There are neurons in the human brain that produces dopamine. Dopamine is a chemical that receives and pass on the information between the substantia nigra and the other parts of the brain which controls the movement of human body. These neurons are accumulated in a particular area of the brain called substantia nigra [8].

In humans, dopamine helps to have smooth coordination in muscles. Symptoms of PD are depression, postural disturbance, hallucinations, tremor in one arm, leg, one side of the body, lips, chin and tongue, sleep distruptions, urinary problems, constipation, difficult to swallow food and speak, improper walking, weakness in voice (shaky rough breathy voice), gastrointestinal disturbance ,Stiffness in the muscles. PD impairs the quality of life. To diagnose PD different signals have been used such as speech, EEG signal, and images like MRI (Magnetic Resonance Imaging) and CT(computed tomography) . Voice analysis is used to detect illnesses in the PD. Diagnosis is based on examination of the neurological behaviour and medical history by observing the patients [10]. After PD, the most common brain disease is Alzheimer disease. It is the cause of dementia which includes memory loss, not able to think or memorize things. In this system, EEG signals and speech signals have been used to detect different brain diseases such as PD, Alzheimer disease where features were extracted for EEG signal and speech signal and SVM is used for classification.

\section{RELATED WORK}

Marius Ene[2] introduces application of three probabilistic neural network (PNN) such as incremental search(IS), Monte Carlo search (MCS)and hybrid search(HS) to distinguish between healthy and unhealthy people with Parkinson's disease. Saloni[4] used voice features with artificial neural network(ANN) and support vector machines(SVM), quadratic and linear classification method for diagnosing Parkinson disease. Tremor is the basic symptom of PD and it is observed in jitter and shimmer values. People suffering with PD show higher jitter values. A.M. Ardi Handojoseno.et.al[5] used multiresolution decomposition of EEG signal and total wavelet entropy and EEG subbands were extracted. The freezing of PD patient is identified by back propagation neural network. James M.Shine.et.al[6] introduced multilayer perceptron neural network and $\mathrm{k}$ nearest neighbour classification method for integration of spatial, spectral and temporal features of EEG signals. Ingeborg H. Hansen.et.al [7] studied classification method of EEG with two classes control subjects and iRBD. Two different classifiers $k$ means and Bayesian is used. Mohammad Shahbakhi.et.al[8] proposed an algorithm and used voice analysis for detection of PD. Genetic algorithm(GA) is used to select optimized features. Support vector machine is used as a classifier. Max A. Little.et.al[9] worked on dysphonia measures to distinguish between healthy people and people suffering from PD. A new factor of dysphonia pitch period entropy (PPE) was introduced. Mohammad Shahbakhti.et.al[10] studied that PD could be detected by many signals such as speech , gait, EEG signal. It proposes a new algorithm where PCA was used and SVM method was used for classification. R. Arefi Shirvan.et.al[11] using voice signals, features were extracted from normal people and people infected with PD. Genetic algorithm was used to detect features and KNN classification method was used. Gertrud Laura Sorensen.et.al[13] studied to propose an algorithm for detection of arousals from sleep . Artificial neural network (ANN) is used as a classifier.

\section{METHODOLOGY FOR EEG SIGNAL}

\subsection{Pre-Processing}

In this, pre-processing of EEG signal was done using zero low pass filter (ZLPF). Low pass filters provide smoothing of signal, removes noise and fluctuations. Features in the filtered waveform are preserved by zero low pass filtering. Zero low pass filtering helps to remove baseline in original EEG signal.

The next part of pre-processing is notch filter designing. Notch filter is a bandstop filter having two passbands and a 
narrow stopband. Use of notch filters attenuates frequency from the input spectrum while the amplitude of the other frequencies remains unchanged. It removes noise and power line interferences in EEG signal.

\subsection{Feature Extraction}

Feature extraction is method of reducing dimension of a large input data. When input data which is to be used is very large but does not has too much information, it will be transform the data into a set of features which is also known as feature vectors [16]. Useful content of EEG signal is provided in frequency domain representation rather than time domain. EEG represents a non-stationary signal thus discrete wavelet transform (DWT) is well suited for it as it provides multiresolution decomposition [16]. At high frequencies good time resolution and at low frequencies better frequency resolution is provided by DWT. Different DWT wavelet are available for feature extraction but Daubechies, Symlets have been used as it provides better results[19].

The dilation parameter $\varphi_{\mathrm{i}, \mathrm{k}}(\mathrm{n})$ is based on low pass filter and wavelet parameter $\psi_{\mathrm{i}, \mathrm{k}}(\mathrm{n})$ is based on high pass filter which is represented as follows:

$$
\begin{aligned}
& \varphi_{\mathrm{i}, \mathrm{k}}=2^{\mathrm{i} / 2} \mathrm{~h}\left(2^{\mathrm{i}} \mathrm{n}-\mathrm{k}\right) \\
& \psi_{\mathrm{i}, \mathrm{k}}=2^{\mathrm{i} / 2} \mathrm{~g}\left(2^{\mathrm{i}} \mathrm{n}-\mathrm{k}\right)
\end{aligned}
$$

The aim of DWT is decomposing the observed EEG signal into detailed coefficient subset(Dj) and approximate coefficient(Aj) subset till the level $\mathrm{j}$ are as follows [22].

$$
\begin{aligned}
& \mathrm{Aj}=\frac{1}{\sqrt{\mathrm{M}}} \sum_{\mathrm{n}} \mathrm{x}(\mathrm{n}) \times \varphi_{\mathrm{i}, \mathrm{k}}(\mathrm{n}) \\
& \mathrm{Dj}=\frac{1}{\sqrt{\mathrm{M}}} \sum_{\mathrm{n}} \mathrm{x}(\mathrm{n}) \times \psi_{\mathrm{i}, \mathrm{k}}(\mathrm{n})
\end{aligned}
$$

$\mathrm{k}=0,1,2 \ldots ., 2^{\mathrm{i}}-1$, where $\mathrm{M}$ denotes length of EEG signal. Relative and Total wavelet energy is,

At each decomposition level $\mathrm{j}=1, \ldots, \mathrm{L}$ is given as follows :

$$
\begin{aligned}
& E_{D_{j}}=\sum_{i=1}^{N}\left|D_{j i}\right|^{2}, \quad j=1,2 \ldots . L \\
& E_{A_{j}}=\sum_{i=1}^{N}\left|A_{j i}\right|^{2}, \quad j=L
\end{aligned}
$$

The maximum level of decomposition is denoted by L. Total energy can be formulated as:

$$
\mathrm{E}_{\text {Total }}=\left(\sum_{\mathrm{j}=1}^{\mathrm{L}} \mathrm{E}_{\mathrm{D}_{\mathrm{j}}}+\mathrm{E}_{\mathrm{A}_{\mathrm{L}}}\right)
$$

Relative wavelet energy is represented by normalized energy values.

$$
\mathrm{E}_{\mathrm{r}}=\frac{\mathrm{E}_{\mathrm{i}}}{\mathrm{E}_{\text {Total }}}
$$

Where,

$$
E_{i}=E_{D_{j=1 \ldots L}} \text { or } E_{A_{i=L}}
$$

Using EEG signal 13 features were extracted, where Daubechies wavelet was used for energy calculation and Symlet wavelet was used for calculating statistical features which are defined as follows,

Mean: Mean is the average value of the numbers. It is the central value of the set of numbers.

Kurtosis: It is the measure of the tailedness of the probability distribution function of a real valued random variable. It is formulated as:

Kurtosis $=\frac{\sum_{\mathrm{i}=1}^{\mathrm{N}}\left(\mathrm{Y}_{\mathrm{i}}-\overline{\mathrm{Y}}\right)^{4} / \mathrm{N}}{\mathrm{s}^{4}}$

Where, $\overline{\mathrm{Y}}$ is the mean,s is standard deviation and $\mathrm{N}$ is the number of data points.

Skewness: It is a measure of the asymmetry of the probability distribution of a real-valued random variable. It can be positive, negative, or even undefined.

$$
\text { Skewness }=\frac{\sum_{\mathrm{i}=1}^{\mathrm{N}}\left(\mathrm{Y}_{\mathrm{i}}-\overline{\mathrm{Y}}\right)^{3} / \mathrm{N}}{\mathrm{s}^{3}}
$$

Median: It is the value that separates the higher half of a data sample or a probability distribution from the lower half of the data sample.

Mode: It is the value that appears most often in the set of data.

Standard deviation: It is the measure that is used to quantify the amount of variation or dispersion of a set of data. It is formulated as:

$$
\sigma=\sqrt{\frac{\sum(\mathrm{x}-\mu)^{2}}{\mathrm{~N}}}
$$

Where $\sigma$ is the entire population, $\mathrm{x}$ is the each value in the population, $\mu$ is the mean and $\mathrm{N}$ is the number of values.

Range: It is the difference between the largest and the smallest values of the set of data.

\subsection{Classifier: Support Vector Machine}

Electroencephalography (EEG) signals which are the neural activities of brain are classified using the most popular machine learning technique called as Support Vector Machine (SVM). For analyzing and interpreting the characteristics of brain activities, EEG signals are mapped into high dimensional feature space, in which kernel functions are used for non linear mapping. The main aim of SVM is to maximize the margin to reduce over fitting and misclassification error of data [18].

SVM is used for construction of hyperplane with large margin to divide data into two groups. Linearly separable classification is used which divides the high dimensional data into two groups,

$$
y_{\mathrm{i}}=\{+1,-1\}
$$

To minimize objective, the function is given as:

$$
\frac{1}{\mathrm{~m}} \sum_{\mathrm{i}=1}^{\mathrm{m}} \mathrm{l}\left(\mathrm{f}\left(\mathrm{x}_{\mathrm{i}}, \alpha\right), \mathrm{y}_{\mathrm{i}}\right)+\text { complexity term }
$$

Where, $f(x)=(w \cdot x)+b$

$$
\frac{1}{m} \sum_{i=1}^{m} l\left(f\left(w \cdot x_{i}+b\right), y_{i}\right)+\|w\|^{2}
$$


Minimizing $\|\mathrm{w}\|^{2}$,

$$
\begin{aligned}
& \left(w \cdot x_{i}+b\right) \geq 1, \quad \text { if } y_{i}=1 \\
& \left(w \cdot x_{i}+b\right) \leq-1, \quad \text { if } y_{i}=-1
\end{aligned}
$$

The quadratic program of SVM is given as,

$$
\mathrm{y}_{\mathrm{i}}\left(\mathrm{w} \cdot \mathrm{x}_{\mathrm{i}}+\mathrm{b}\right) \geq 1
$$

Support vector machines (SVM) proposes a model which uses the training set of EEG signals where each one is marked to its category and then classified. In SVM classifier, hyperplane which represents the largest separation amongst the three classes is selected. Maximum margin hyperplane is a plane where distance to the nearest data point on both sides is maximized. In every cases, maximum margin hyperplane is selected [4].

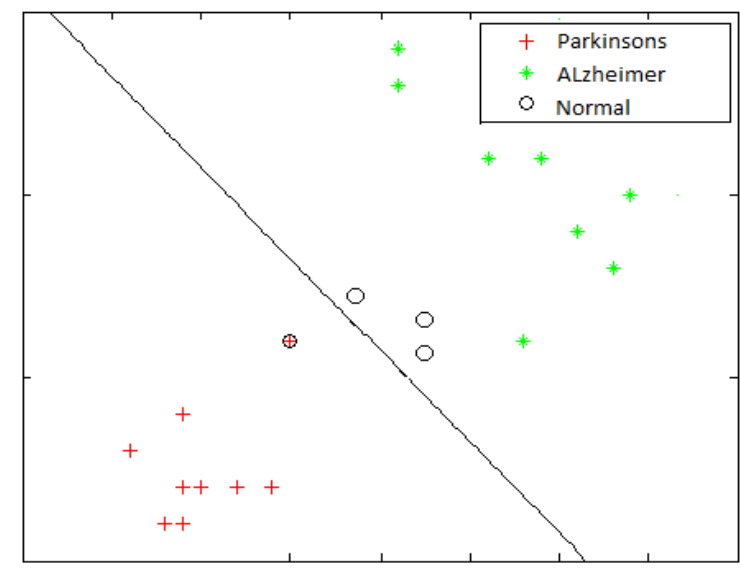

Fig 1: SVM Classification

Using SVM classification, different brain diseases were classified such as Parkinson's disease, Alzheimer disease and people which are healthy.

\section{PROPOSED METHODOLOGY FOR SPEECH SIGNALS}

Speech is the easiest way of communication for humans. Speech signals occur naturally so they are random signals. Speech recognition system is the method of recognizing word automatically spoken by a person which is based on information present in the sample of voice. The basics steps of speech recognition system is speech sample, denoising, feature extraction, pattern machine. It trains the system so that it can recognize voice characteristics of a person [23].

\subsection{Pre-Processing}

In Pre-processing, the denoising of speech signal is done by settling a particular threshold value, the part below threshold is considered as the silence part and the part above threshold is consider as speech input signal of the feature extraction stage.

\subsection{Feature Extraction}

For MFCCs are the coefficients obtained in the MFC (mel frequency cepstrum) representation. Mel frequency Cepstral Coefficients (MFCC) is very efficient feature extraction method which is less complex when implemented and under various different conditions it shows robustness [23].

- Pre-Emphasis: In this process high frequencies are emphasized when the word sample is passed through the filter which also increases the energy of the signal[23].
- Frame Blocking: Framing is necessary because speech signal is a time varying signal but when viewed over a period of time it appears like a stationary signal. Speech signal is divided into small duration blocks called frames [23].

- Hamming Windowing: In this process, the continuity of the signal is maintained by multiplying the frames with the hamming window [23].

- Fast Fourier Transform (FFT): The process of conversion of time domain into frequency domain is called as FFT. Magnitude frequency response of each particular frame is obtained by performing FFT and we get spectrum as the output [23].

- Triangular band pass filters: To get smooth magnitude spectrum, the magnitude frequency response is multiplied by 20 triangular band pass filters [23].

$$
\text { Mel }(f)=1125^{*} \ln (1+f / 700)
$$

- Discrete Cosine Transform(DCT): To obtain L mel-scale cepstral coefficients we apply discrete cosine transform which is formulated as follows:

$$
\mathrm{C}_{\mathrm{m}}=\sum_{\mathrm{k}=1}^{\mathrm{N}} \cos \left[\mathrm{m} *(\mathrm{k}-0.5) \frac{\pi}{\mathrm{N}}\right] * \mathrm{E}_{\mathrm{k}}
$$

where $\mathrm{m}=1,2 \ldots \mathrm{L}$

- Delta Energy: Energy is calculated within a frame which is an another feature to MFCC.

\subsection{Classifier:Minimum Distance Classifier}

In Minimum distance classifier works, when the distance between mean is large to the spread or randomness of each class with respect to its mean. It yields optimum performance in terms of minimizing the average loss of misclassification when the distribution of each class about its mean is in the form of a spherical. It is computationally simple and fast.

In minimum distance classifier, the mean of the spectral values for the training set in each band is calculated. It measures distance from the pixel to the mean of each category. It assigns the pixels to the category with shortest distance. If the pixel is beyond the distance it assigns the pixel as unknown.

Training: Each class prototype (mean) is calculated.

Classification: Assigns the unlabeled patterns to the nearest prototype in the feature space

The basic idea of minimum distance classifier is not to know the distances, but to find which one is the closest.

The minimum distance classifier is formulated as:

$\left\|\mathrm{x}-\mathrm{m}_{\mathrm{i}}\right\|$

Using Minimum distance classifier, different diseases such as Alzheimer, PD, and normal people were detected.

\section{RESULTS}

To classify between healthy normal people and people suffering from different brain diseases such as Parkinson's and Alzheimer. Various different features were extracted using discrete wavelet transform (DWT). In order to 
implement SVM classification training and testing methods were applied, MATLAB software is used. For evaluating the performance, three statistical parameters such as sensitivity, specificity, total classification accuracy is calculated as follows:

$$
\begin{aligned}
& \text { Specificity for PD }=\frac{\text { NCPD }}{\text { NTPD }} \times 100 \\
& \text { Specificity for AD }=\frac{\text { NCAD }}{\text { NTAD }} \times 100 \\
& \text { Sensitivity }=\frac{\text { NCH }}{\text { NTH }} \times 100 \\
& \text { Total Classification Accuracy }=\frac{\text { NCCP }}{\text { NTP }} \times 10
\end{aligned}
$$

where NCPD and NCAD is number of correctly classified Parkinson's disease (PD) and Alzheimer disease(AD), NTPD and NTAD is number of total PD and $\mathrm{AD}, \mathrm{NCH}$ is number of correctly classified people and NTH is number of total persons.

\section{Table 1.Three Statistical Parameters for Proposed Method}

\begin{tabular}{|l|l|l|l|l|}
\hline $\begin{array}{l}\text { Type of } \\
\text { Signal }\end{array}$ & $\begin{array}{l}\text { Statistical } \\
\text { Parameters }\end{array}$ & Specificity & Sensitivity & $\begin{array}{l}\text { Total } \\
\text { Accuracy }\end{array}$ \\
\hline $\begin{array}{l}\text { EEG } \\
\text { Signal }\end{array}$ & PD & $100 \%$ & $75 \%$ & $91.6 \%$ \\
\cline { 2 - 5 } & AD & $75 \%$ & $75 \%$ & $75 \%$ \\
\hline \multirow{2}{*}{$\begin{array}{l}\text { Speech } \\
\text { Signal }\end{array}$} & PD & $100 \%$ & $100 \%$ & $100 \%$ \\
\cline { 2 - 5 } & AD & $100 \%$ & $100 \%$ & $100 \%$ \\
\hline
\end{tabular}

\section{CONCLUSION}

The aim of this paper is to detect different brain diseases such as Parkinson's disease, Alzheimer disease using EEG and speech signal. In this paper the EEG dataset consists of 20 people where 8 people suffered from PD, 8 with Alzheimer and 4 people were healthy and Speech signal dataset consists of 70 people where 30 people were detected with Parkinson's, 30 people with Alzheimer and 10 people were detected healthy. Different features were extracted using DWT and classification was done using SVM network and accuracy obtained was $91.6 \%$. It is concluded that EEG and speech signals of brain disease patient provides the basis for detecting different brain diseases and the technique developed in this study shows promising results.

\section{FUTURE SCOPE}

Using EEG and Speech signals different brain diseases can be detected, but also using Gait analysis brain diseases like Alzheimer disease, PD can also be detected. Principal Component Analysis (PCA) algorithm can also be applied for feature extraction. Artificial neural network techniques can also be implemented for detecting and classifying healthy and people suffering from different brain diseases.

\section{REFERENCES}

[1] Chunchu Rambabu, B Rama Murthy," EEG Signal with Feature Extraction using SVM and ICA Classifiers", International Journal of Computer Applications (0975 8887)Volume 85 - No 3, January 2014.

[2] Marius Ene," Neural network-based approach to discriminate healthy people from those with Parkinson's disease", Annals of the University of Craiova, Math. Comp. Sci. Ser.Volume 35, 2008
[3] Patrizio Campisi, Senior Member, IEEE, and Daria La Rocca, Student Member, IEEE," Brain Waves for Automatic Biometric-Based User Recognition", IEEE transactions on information forensics and security, vol. 9, no. 5, may 2014 .

[4] Saloni, R. K. Sharma and Anil K. Gupta," Artificial Neural Networks and Support Vector Machines for Parkinson Disease Detection using Human Voice", Advances in Circuits, Systems, Signal Processing and Telecommunications 2015

[5] A.M. Ardi Handojoseno, James M. Shine, Tuan N. Nguyen, Member, IEEE, Yvonne Tran, Simon J.G. Lewis, Hung T. Nguyen, Senior Member, IEEE," The detection of Freezing of Gait in Parkinson's disease patients using EEG signals based on Wavelet Decomposition",34thAnnual International Conference of the IEEE EMBS San Diego, California USA, 28 August - 1 September, 2012

[6] A.M. Ardi Handojoseno, James M. Shine, Tuan N. Nguyen, Member, IEEE, Yvonne Tran, Simon J.G. Lewis, Hung T. Nguyen, Senior Member, IEEE," Using EEG Spatial Correlation, Cross Frequency Energy, and Wavelet Coefficients for the prediction of Freezing of Gait in Parkinson's disease patients",35th Annual International Conference of the IEEE EMBS Osaka, Japan, 3 - 7 July, 2013.

[7] Ingeborg H. Hansen, Mikkel Marcussen, Julie A. E. Christensen, Poul Jennum, and Helge B. D. Sorensen,' Detection of a Sleep Disorder Predicting Parkinson's Disease",35th Annual International Conference of the IEEE EMBS Osaka, Japan, 3 - 7 July, 2013.

[8] Mohammad Shahbakhi, and Danial Taheri Far and Ehsan Tahami2, "Speech Analysis for Diagnosis of Parkinson's Disease Using Genetic Algorithm and Support Vector Machine",J. Biomedical Science and Engineering, 2014, 7, 147-156.

[9] Max A. Little, Patrick E. McSharry, Eric J. Hunter, Jennifer Spielman, and Lorraine O. Ramig," Suitability of Dysphonia Measurements for Telemonitoring of Parkinson's Disease", IEEE transactions on biomedical engineering, vol. 56, no. 4, april 2009.

[10] Mohammad Shahbakhti, Danial Taherifar, Zahra Zareei," Combination Of PCA And SVM For Diagnosis Of Parkinson's Disease", 2nd International Conference on Advances in Biomedical Engineering,2013.

[11] R. Arefi Shirvan, E. Tahami," Voice Analysis for Detecting Parkinson's Disease Using Genetic Algorithm and KNN Classification Method", 18th Iranian Conference on BioMedical Engineering, 14-16 December 2011, Tehran, Iran.

[12] Sabato Santaniello, David L. Sherman, Nitish V. Thakor, "Optimal Control-based Bayesian detection of clinical ", IEEE transactions on neural systems and rehabilitation engineering, vol. 20, no. 5, september 2012.

[13] Gertrud Laura Sorensen, Jacob Kempfn, Poul Jennum and Helge B. D. Sorense, "Detection of arousals in Parkinson's disease patients",33rd Annual International Conference of the IEEE EMBS Boston, Massachusetts USA, August 30 - September 3, 2011 
[14] H.H.Stassen," Computerized recognition of persons by EEG spectral patterns",Electroencephalography and ClinicalNeurophsiology,1980,49:190-194,

Elsevier/North-Holland Scientific Publishers, Ltd.

[15] Lucas R. Trambaiolli, Tiago H. Falk, Francisco J. Fraga, Renato Anghinah, and Ana C. Lorena,"EEG SpectroTemporal Modulation Energy : a New Feature for Automated Diagnosis of Alzheimer's Disease",33rd Annual International Conference of the IEEE EMBSBoston, Massachusetts USA, August 30 September 3, 2011.

[16] Ashwini Nakate, P.D. Bahirgonde," Feature Extraction of EEG Signal using Wavelet Transform",International Journal of Computer Applications (0975 - 8887)Volume 124 - No.2, August 2015

[17] M.A.Anusuya, S.K.Katti," Speech Recognition by Machine: A Review",(IJCSIS) International Journal of Computer Science and Information Security,Vol. 6, No. $3,2009$.

[18] P Bhuvaneswari, J Satheesh Kumar," Support Vector Machine Technique for EEG Signals",International Journal of Computer Applications (0975 - 8887) Volume 63- No.13, February 2013.

[19] Mohammad H. Alomari, Emad A. Awada, Aya Samaha1 and Khaled Alkamh," Wavelet-Based Feature Extraction for the Analysis of EEG Signals associated with Imagined Fists and Feet Movements", Computer and Information Science; Vol. 7, No. 2,2014.
[20] Alan W.C. Tan, M.V.C. Rao, B.S. Daya Sagar,"A signal subspace approach for speech modelling and classification”, ELSEVIER Signal Processing 87 (2007) 500-508,2006.

[21] Monica Mundada, Sangramsing Kayte, Sumegh Tharewal, Dr Bharti Gawali,"Classification of Fluent and Dysfluent Speech using KNN classifier",International Journal of Advanced Research in Computer Science and Software Engineering 4(9), September - 2014, pp. 1-7.

[22] Hafeez Ullah Amin and Aamir Saeed Malik and Rana Fayyaz Ahmad." Feature extraction and classification for EEG signals using wavelet transform and machine learning techniques", Australasian College of Physical Scientists and Engineers in Medicine,SPRINGER,2015.

[23] Parwinder Pal Singh and Pushpa Rani," An Approach to Extract Feature using MFCC",IOSR Journal of Engineering (IOSRJEN)Vol. 04, Issue 08 (August. 2014).

[24] Siddhant C. Joshi and Dr. A.N.Cheeran," MATLAB Based Feature Extraction Using Mel Frequency Cepstrum Coefficients for Automatic Speech Recognition",International Journal of Science, Engineering and Technology Research (IJSETR), Volume 3, Issue 6, June 2014 